\title{
Is the silicate emission feature only influenced by grain size?`
}

\author{
N. V. Voshchinnikov ${ }^{1,2}$ and T. Henning ${ }^{3}$ \\ ${ }^{1}$ Sobolev Astronomical Institute, St. Petersburg University, Universitetskii prosp. 28, St. Petersburg 198504, Russia \\ e-mail: nvv@astro.spbu.ru \\ 2 Isaac Newton Institute of Chile, St. Petersburg Branch, Russia \\ 3 Max-Planck-Institut für Astronomie, Königstuhl 17, 69117 Heidelberg, Germany
}

Received 3 March 2008 / Accepted 21 March 2008

\section{ABSTRACT}

\begin{abstract}
The flattening of the $10 \mu \mathrm{m}$ silicate emission feature observed in the spectra of T Tauri and Herbig Ae/Be stars is usually interpreted as an indicator of grain growth. We show in this paper that a similar behaviour of the feature shape occurs when the porosity of composite grains varies. We modelled the fluffy aggregates with inclusions of different sizes with multi-layered spheres consisting of amorphous carbon, amorphous silicate, and vacuum. We also found that the inclusion of crystalline silicates in composite porous particles can lead to a shift of the known resonances and production of new ones.
\end{abstract}

Key words. line: profiles - stars: pre-main-sequence - stars: planetary systems: protoplanetary disks - stars: circumstellar matter infrared: stars - ISM: dust, extinction

\section{Introduction}

The shape and strength of the silicate emission feature observed near $10 \mu \mathrm{m}$ in the spectra of T Tauri and Herbig Ae/Be (HAeBe) stars is commonly used as a measure of grain growth in protoplanetary discs (see Natta et al. 2007, for a review). It is wellknown theoretically that with an increase of the grain size, the feature becomes wider and eventually fades away. In the case of compact spherical grains with a composition of astronomical silicate, the $10 \mu \mathrm{m}$ feature disappears when the grain radius exceeds $\sim 2 \mu \mathrm{m}$ (see Fig. 1).

The standard approach to modelling the $10 \mu \mathrm{m}$ feature includes calculations of light absorption by several populations of compact (and hollow) silicate spheres. Amorphous and crystalline particles of small and large sizes are used to fit the observed emission profiles. The model was first suggested by Bouwman et al. (2001) and then modified by van Boekel et al. (2005). It was used by Schegerer et al. (2006); Honda et al. (2006); Kessler-Silacci et al. (2006); Sargent et al. (2006); Sicilia-Aguilar et al. (2007); and Bouwman et al. (2008) to fit the observational data. Further the authors searched for correlations between the estimated mass fractions of large and crystalline grains and different stellar and disc parameters like mass, luminosity, age, spectral type, etc. As a rule, the correlations are absent or rather weak (see Table 5, Sicilia-Aguilar et al. 2007).

In this Letter, we show that the shape, position, and strength of the $10 \mu \mathrm{m}$ silicate feature is also influenced by variations of the properties of small mass composite aggregate grains. We modelled the fluffy aggregates by multi-layered spheres (see also Voshchinnikov et al. 2006). This particle model permits us to include an arbitrary fraction of materials, and computations do not require large resources.

* Table 1 is only available in electronic form at http: //www . aanda.org

\section{Model of porous composite grains}

Fluffy particles should appear as a result of grain coagulation in interstellar clouds and protoplanetary discs (e.g., Henning \& Stognienko 1996; Dominik \& Tielens 1997; Jones 2004; Ormel et al. 2007). It is expected that aggregates can consist of several silicate and carbon sub-particles of different sizes that can be treated as inclusions in a vacuum matrix.

We use the model of spherical multi-layered particles introduced by Voshchinnikov \& Mathis (1999). Later, Voshchinnikov et al. (2005) demonstrated that the optical properties of layered spheres resemble those of fluffy aggregates with inclusions of different sizes.

Our model parameters are: the refractive indices and volume fractions $V_{\mathrm{i}} / V_{\text {total }}$ of the materials and the radius of compact particles $r_{\text {compact }}$. The amount of vacuum in the particle (the particle porosity $\mathcal{P}, 0 \leq \mathcal{P}<1$ ) is

$\mathcal{P}=V_{\text {vac }} / V_{\text {total }}=1-V_{\text {solid }} / V_{\text {total }}$,

where $V_{\text {solid }}$ is the sum of the volumes of all species excluding vacuum. The radius of porous particles is related to that of compact particles as

$r_{\text {porous }}=\frac{r_{\text {compact }}}{(1-\mathcal{P})^{1 / 3}}=\frac{r_{\text {compact }}}{\left(V_{\text {solid }} / V_{\text {total }}\right)^{1 / 3}}$.

The model of layered spheres combines all components, including vacuum in one particle (internal mixing) in contrast to the standard approach discussed above where "external mixing" (mixture of several individual populations of compact grains) is used.

For calculations, we use different kinds of carbon and silicates: amorphous carbon Be1 and AC1 (Rouleau \& Martin 1991), amorphous silicate with olivine composition (Dorschner et al. 1995), crystalline olivine (Fabian et al. 2001), and astronomical silicate (astrosil; Laor \& Draine 1993). 


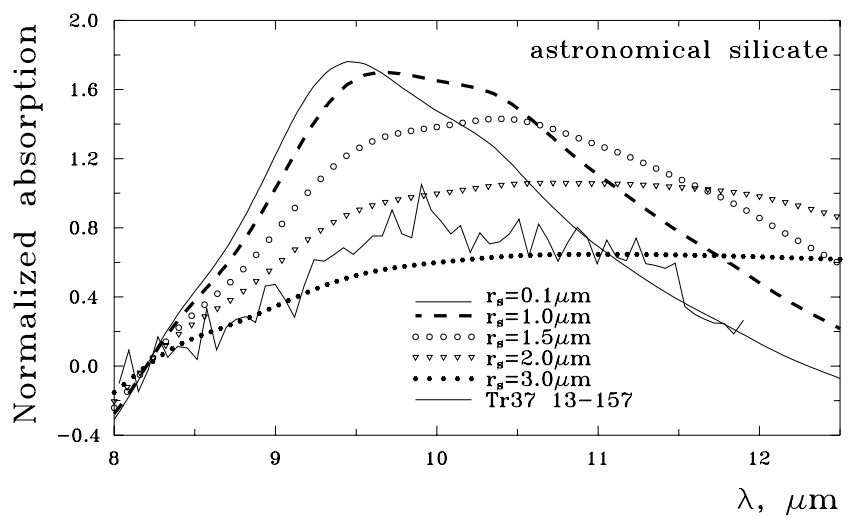

Fig. 1. Wavelength dependence of the normalised absorption efficiency factor $\left[Q_{\mathrm{abs}}(\lambda) / Q_{\mathrm{abs}}(8.2 \mu \mathrm{m})-1\right]$ for compact spheres consisting of astrosil. The effect of variation of the silicate emission shape with the particle size is illustrated. The observational profile of the T Tauri star Tr 37 13-157 (Sicilia-Aguilar et al. 2007) is shown for comparison. Note that this profile is not continuum subtracted.

\section{Analysis of silicate emission}

The silicate feature in the $N$ band was observed in spectra of a large variety of objects (see Henning 2008, for a recent review). We should caution the reader that we calculate absorption efficiencies, but measure the fluxes. A detailed analysis of disc spectra certainly requires radiative transfer calculations. Assuming that the silicate emission is optically thin, we can compare observed fluxes with theoretical profiles. The latter are proportional to the product of particle absorption cross section by the Planck function with the particle temperature $\propto C_{\text {abs }}(\lambda) B_{\lambda}\left(T_{\mathrm{d}}\right)$. Radiative transfer calculations show that grains of different temperatures contribute to the silicate feature (see Fig. 1 in Schegerer et al. 2006). However, the dominant contribution for the $10 \mu \mathrm{m}$ feature comes from particles with $T_{\mathrm{d}}=$ 200-400 K for which the Planck function is approximately constant in $N$ band. Therefore, we can adopt that the shape of the feature depends primarily on the emission properties of grains ${ }^{1}$.

The profile of the feature can be described by the normalised absorption efficiency factor $Q_{\mathrm{abs}}(\lambda) / Q_{\mathrm{abs}}\left(\lambda_{0}\right)-1$, where the flux at wavelength $\lambda_{0}$ characterises the continuum. As usual, the value of $\lambda_{0}=8.2 \mu \mathrm{m}$ is chosen (e.g., Schegerer et al. 2006).

Another representation of the optical behaviour is provided by the mass absorption coefficient, which is the ratio of absorption cross section $C_{\text {abs }}(\lambda)$ to particle mass. In the case of a sphere of porosity $\mathcal{P}$, it can be written as

$\kappa_{\mathrm{abs}}(\lambda)=\frac{C_{\mathrm{abs}}(\lambda)}{\rho_{\mathrm{d}} V_{\text {total }}}=\frac{3 Q_{\mathrm{abs}}(\lambda)}{4 \rho_{\mathrm{d}, \text { solid }} r_{\mathrm{s}, \text { compact }}(1-\mathcal{P})^{2 / 3}}$,

where $\rho_{\mathrm{d}}=\rho_{\mathrm{d} \text {, solid }}(1-\mathcal{P})$ is the mean particle density. The value of $\rho_{\mathrm{d} \text {, solid }}$ is obtained by averaging the density of all species excluding vacuum. In our calculations, we assume that $\rho_{\mathrm{d}, \mathrm{Si}}=3.3 \mathrm{~g} / \mathrm{cm}^{3}$ and $\rho_{\mathrm{d}, \mathrm{C}}=1.85 \mathrm{~g} / \mathrm{cm}^{3}$ for silicate and carbon, respectively.

Figure 1 shows the wavelength dependence of the normalised absorption efficiency factors for compact silicate spheres of diverse sizes. With a growth of the particle size (and mass), the $10 \mu \mathrm{m}$ silicate feature broadens, its height decreases and the position of maximum shifts to longer wavelengths.

\footnotetext{
${ }^{1}$ Note that our case differs from the case analysed by Li et al. (2004) where the cometary particles of different composition are located at the same distance from the Sun.
}
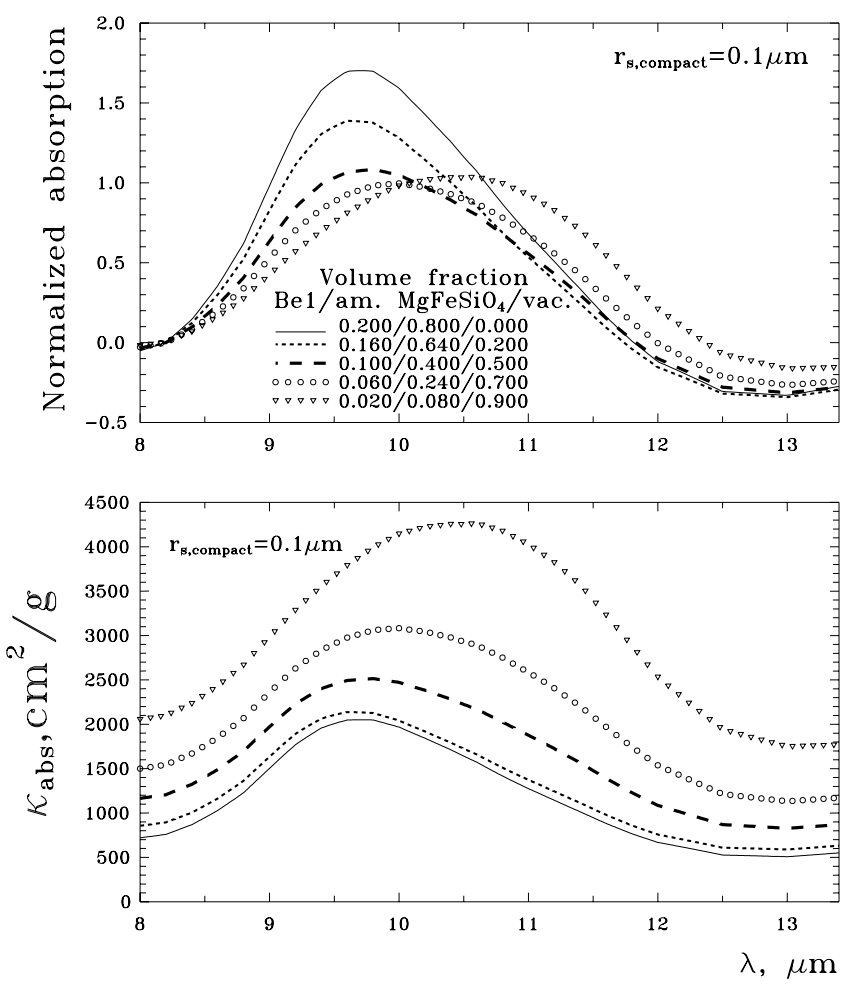

Fig. 2. Wavelength dependence of the normalised absorption efficiency factor (upper panel) and mass absorption coefficient (lower panel) for layered spheres with $r_{\mathrm{s} \text {, compact }}=0.1 \mu \mathrm{m}$. The particles consist of amorphous carbon (Be1), amorphous silicate with olivine composition $\left(\mathrm{MgFeSiO}_{4}\right)$, and vacuum. The volume fraction of components $V_{i} / V_{\text {total }}$ is indicated. The particles are of the same mass $\left(V_{\mathrm{Be} 1} / V_{\text {solid }}=\right.$ $\left.V_{\mathrm{Be} 1} /\left(V_{\mathrm{Be} 1}+V_{\mathrm{MgFeSiO}_{4}}\right)=0.2\right)$, but of different porosity. The effect of variation of the silicate emission shape with the particle porosity is illustrated.

In a similar manner, the silicate feature changes when the particle porosity grows (Fig. 2). However, in this case the particle size increases only moderately, while particle mass remains the same. With a growth of porosity, the peak strength decreases for normalised absorption (Fig. 2, upper panel) whereas the mass absorption coefficient becomes larger (Fig. 2, lower panel). The value of $\kappa_{\text {abs }}$ almost doubles at the peak position when we replace the compact particle by porous particle.

It is well known that the shape and strength of the silicate feature depend on the type of the amorphous silicate, particle size, and fractal dimension (see, e.g., van Boekel et al. 2005; Schegerer et al. 2006; Min et al. 2006). Using the model of composite grains we can also investigate how carbon embedded in particles affects the characteristics of the silicate feature.

This dependence is plotted in Fig. 3. With addition of carbon, the feature very rapidly transforms into a plateau and then disappears.

Comparing the data presented in Figs. 1 and 3 with observations, it is possible to estimate characteristics of grains fitting the observations: $r_{\mathrm{s} \text {, compact }} \approx 2.0 \mu \mathrm{m}$ or $V_{\mathrm{Be} 1} / V_{\text {solid }} \approx 0.2$ and $r_{\mathrm{s} \text {, porous }} \approx 0.215 \mu \mathrm{m}$ (last value is obtained from Eq. (2) for $\mathcal{P}=0.9)$. Hence the same observational data can be explained with particles whose radii differ by a factor of $\sim 9$ and masses by a factor of $\sim 8800($ !).

Using the observations from Schegerer et al. (2006); van Boekel et al. (2005); Sargent et al. (2006); and Sicilia-Aguilar et al. (2007), we fitted observational and theoretical profiles of the silicate feature. We choose the best 


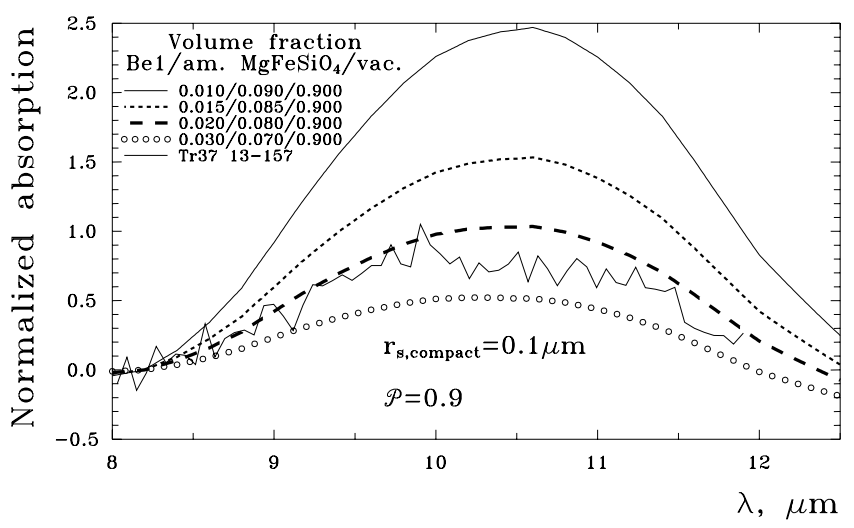

Fig. 3. The same as in Fig. 2 (upper panel) but now for particles of porosity $\mathcal{P}=0.9$. The particles are of the same porosity but of slightly different mass. The value of $V_{\mathrm{Be} 1} / V_{\text {solid }}$ varies from 0.1 to 0.3 . The effect of variation of the silicate emission shape with the volume fraction of carbon is illustrated. The observational profile of the T Tauri star Tr 37 13-157 is shown for comparison.

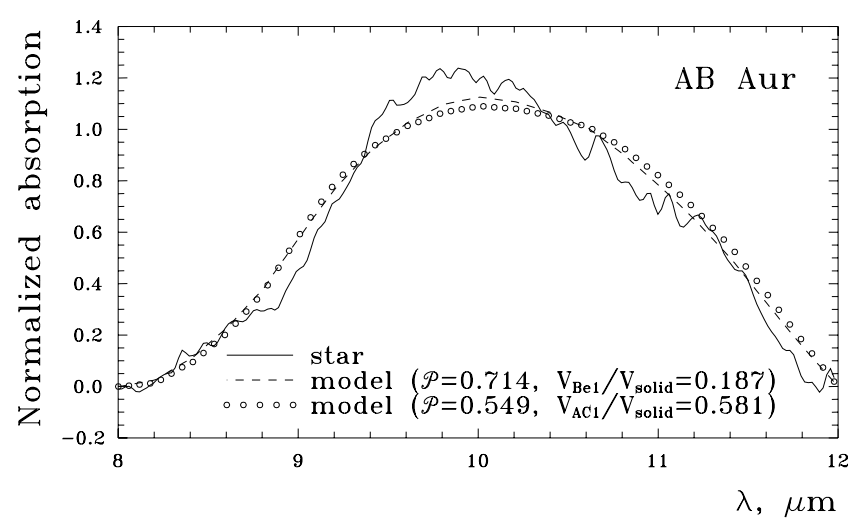

Fig. 4. The observational normalised profile of the $10 \mu \mathrm{m}$ feature for the Herbig Ae/Be star AB Aur (van Boekel et al. 2005; continuum is subtracted). The best fit profiles are shown for particles containing amorphous carbon in the form Be1 (dashed line) and $\mathrm{AC} 1$ (circles). The values of particle porosity $\mathcal{P}$ and fractional amount of carbon $V_{\mathrm{Bel}} / V_{\text {solid }}$ are indicated.

model by minimising the $\chi^{2}$ criterion. In all cases, the observational continuum is fitted by a straight line in the interval $8-12 \mu \mathrm{m}$ for the sake simplicity. We refer the reader to Juhász et al. (2008) for a detailed discussion of the physical models of continuum. We made the calculations for layered spheres with $r_{\mathrm{s}, \text { compact }}=0.1 \mu \mathrm{m}$ consisting of $\mathrm{Be} 1$ and $\mathrm{MgFeSiO}_{4}$.

The estimated porosity and amount of carbon in grains producing the silicate emission feature in protoplanetary discs are collected in online Table 1 . At this stage, the stars with very pronounced crystalline peaks were eliminated from consideration. Table 1 includes 47 stars (30 T Tau and 17 HAeBe stars). The obtained values of $\mathcal{P}, V_{\mathrm{Be} 1} / V_{\text {solid }}$, the ratio of masses of carbon to silicate $M_{\mathrm{Be} 1} / M_{\mathrm{MgFeSiO}_{4}}$ and stellar age (if known) are given. Note that the determination of the age is often quite uncertain for pre-mainsequence stars (see discussion in Blondel \& Tjin A Djie 2006). Therefore, we do not discuss possible correlations.

The grain porosity exceeds 0.5 and the average value of $\mathcal{P}$ is equal to $\langle\mathcal{P}\rangle=0.64 \pm 0.15$. Such particles resemble aggregates obtained both experimentally (Kempf et al. 1999) and theoretically (Shen et al. 2008) ${ }^{2}$. The amount of carbon in grains is not very large (average volume fraction $\left\langle V_{\mathrm{Be} 1} / V_{\text {solid }}\right\rangle=0.24 \pm 0.10$

2 See also discussion in Li et al. (2003).
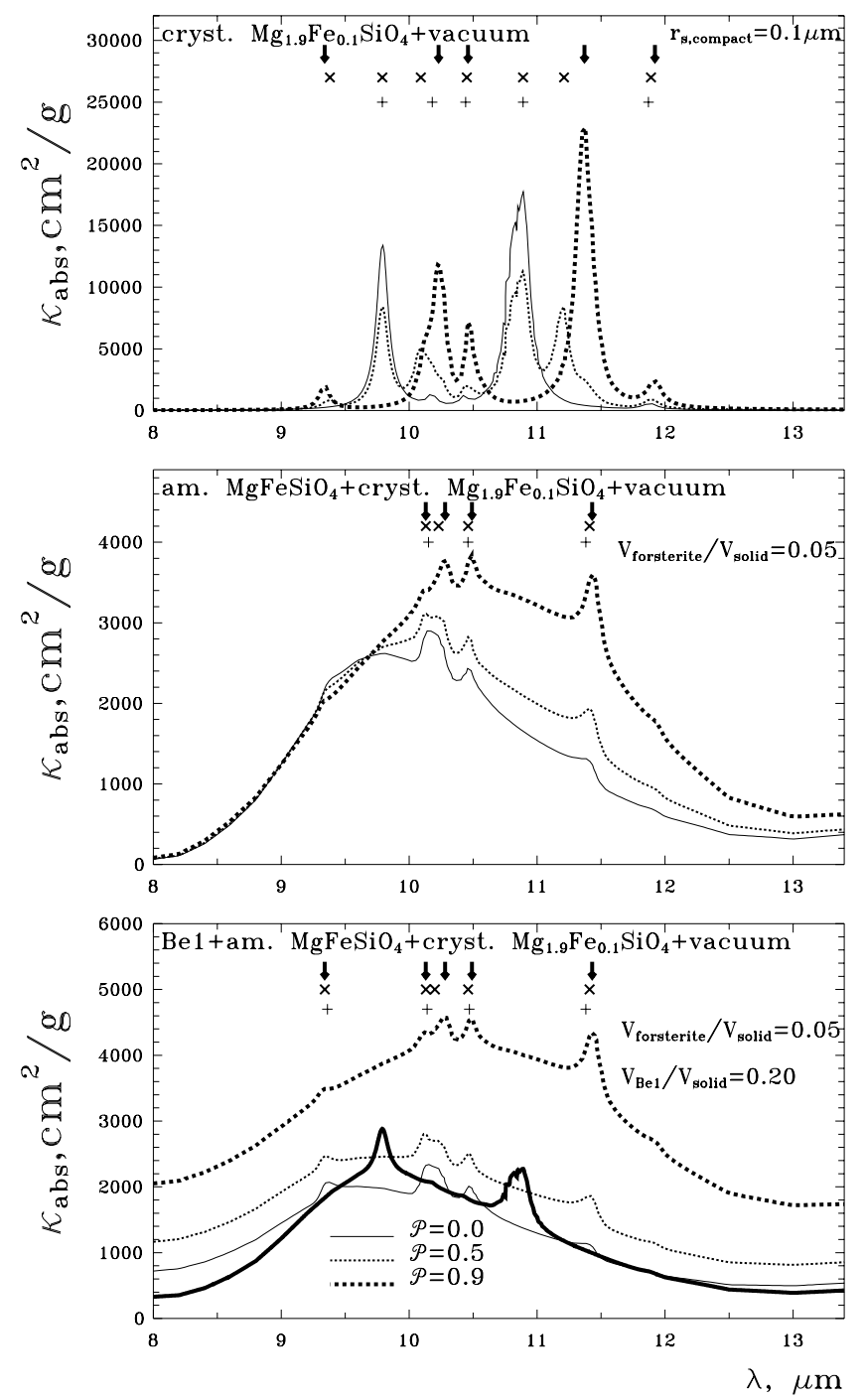

Fig. 5. Wavelength dependence of the mass absorption coefficient for compact and layered spheres with $r_{\mathrm{s}, \text { compact }}=0.1 \mu \mathrm{m}$. The particles consist of crystalline olivine and vacuum (upper panel), amorphous silicate, crystalline olivine, and vacuum (middle panel) and amorphous carbon, amorphous silicate, crystalline olivine, and vacuum (lower panel). The volume fraction of crystalline olivine and $\mathrm{Be} 1$ is indicated. The position of peaks is marked. The thick solid line at the lower panel shows the mass absorption coefficient for the mixture of compact grains (external mixing) with volume fractions of constituents corresponding to solid curve (internal mixing). The effect of variation of crystalline resonances with particle porosity and composition is illustrated.

and average mass ratio $\left\langle M_{\mathrm{Be} 1} / M_{\mathrm{MgFeSiO}_{4}}\right\rangle=0.19 \pm 0.12$ ). These values increase by a factor of $3-4$, if we replace $\mathrm{Be} 1$ by the less absorbing amorphous carbon $\mathrm{AC} 1$. This fact is illustrated in Fig. 4, where the results are given for both particle materials. Note that the particles containing AC1 are less porous.

The variation of grain porosity without significant change of grain mass may explain the behaviour of silicate emission. This explanation is an alternative to the commonly used idea of large grains in protoplanetary discs. We will be able to decide between the two hypotheses after conducting spectropolarimetry in the $10 \mu \mathrm{m}$ feature because a noticeable albedo of large grains manifests itself in polarisation of the scattered light. In this case, we expect unusual behaviour of polarisation parameters (especially positional angle) within the feature profile in comparison with 
calculated profiles for dichroic extinction (see, e.g., Henning \& Stognienko 1993; Prokopjeva \& Il'in 2007).

\section{Crystalline silicates in composite grains}

Another interesting problem is the degree of crystallinity of dust in protoplanetary discs, which is related to the processes of partial grain evaporation and annealing (e.g., Gail 2004). Due to the conversion of amorphous silicates to crystalline minerals, the particles may consist of different types of silicates. In order to show the effect of amorphous silicate matrix and vacuum on resonances produced by crystalline silicates, we calculated the feature profiles for composite particles containing $\mathrm{Mg}$-rich crystalline olivine $\mathrm{Mg}_{1.9} \mathrm{Fe}_{0.1} \mathrm{SiO}_{4}$ as a component. The results are plotted in Fig. 5 where the upper panel illustrates the influence of particle porosity on position and strength of emission peaks. It is seen that variations of spectra are significant: the shape of the feature changes (cf. Fig. 2), some peaks totally disappear and new peaks arise. A very pronounced peak with a maximum near $\lambda=11.37 \mu \mathrm{m}$ is observed for very porous particles whereas compact and medium-porous particles have resonances near $\lambda=10.89 \mu \mathrm{m}$. The inclusion of crystalline silicates in a composite particle containing amorphous silicate (middle panel) or amorphous silicate and carbon (lower panel) changes the picture. The resonance near $\lambda \approx 11.4 \mu \mathrm{m}$ is clearly seen at the long-wavelength wing of the feature. Its position slightly shifts if the porosity changes. Fabian et al. (2001) found that such a peak appeared in the spectra of strongly-elongated particles. A double peaked structure around $\lambda=10.25 \mu \mathrm{m}$ arises as well. This structure was not noticed previously in spectra of crystalline olivines (H. Mutschke, priv. commun., 2007). Note that, as expected, the mixture of separate constituents (amorphous silicate, carbon and crystalline silicate, thick line in lower panel) do not lead to the shift of peaks. Further calculations with different materials for wider wavelength range and detailed comparison with Spitzer observations (see Watson et al. 2007) might help to resolve the problem of grain crystallisation in protoplanetary discs and to answer the question whether the crystals occur in "isolation" or as part of porous grains.

Acknowledgements. We thank R. van Boekel, A. Schegerer, B. Sargent, M. Honda, and A. Sicilia-Aguilar for sending the observational data in tabular form, H. Mutschke, V. Il'in, S. Korneyev, R. van Boekel, A. Juhász and the anonymous referee for helpful comments and suggestions. The work was partly supported by grants NSh 8542.2006.2, RNP 2.1.1.2152 and RFBR 07-02-00831 of the Russian Federation.

\section{References}

Bertout, C., Siess, L., \& Cabrit, S. 2007, A\&A, 473, L21

Blondel, P. F. S., \& Tjin A Djie, H. R. E. 2006, A\&A, 456, 1045

Bouwman, J., Meeus, G., de Koter, A., et al. 2001, A\&A, 375, 950

Bouwman, J., Henning, Th., Hillenbrand, L. A., et al. 2008, ApJ, in press [arXiv: 0802.3033]

Dominik, C., \& Tielens, A. G. G. M. 1997, ApJ, 480, 647

Dorschner, J., Begemann, B., Henning, Th., Jäger, C., \& Mutschke, H. 1995, A\&A, 300, 503

Fabian, D., Henning, Th., Jäger, C., et al. 2001, A\&A, 378, 228

Gail, H.-P. 2004, A\&A, 413, 571

Güdel, M., Briggs, K. R., Arzner, K., et al. 2006, A\&A, 468, 353

Henning, Th. 2008, ESA Publ., in press

Henning, Th., \& Stognienko, R. 1993, A\&A, 280, 609

Henning, Th., \& Stognienko, R. 1996, A\&A, 311, 291

Honda, M., Kataza, H., Okamoto, Y. K., et al. 2006, ApJ, 646, 1024

Jones, A. P. 2004, In Astrophysics of dust, ed. A. N. Witt, G. C. Clayton, \& B. T. Draine, ASP Conf., 309, 347

Juhász, A., Henning, Th., Bouwman, J., et al. 2008, ApJ, in press

Kempf, S., Pfalzner, S., \& Henning, Th. 1999, Icarus, 141, 388

Kessler-Silacci, J. E., Augereau, J.-C., Dullemond, C. P., et al. 2006, ApJ, 639, 275

Laor, A., \& Draine, B. T. 1993, ApJ, 402, 441

Li, A., Lunine, J. I., \& Bendo, G. J. 2003, ApJ, 598, L51

Li, M., Zhao, G., \& Li, A. 2004, ApJ, 613, L145

Min, M., Dominik, C., Hovenier, J. W., et al. 2006, A\&A, 445, 1005

Natta, A., Testi, L., Calvet, N., et al. 2007, In Protostars and Planets V, ed. B. Reipurth, D. Jewitt, \& K. Keil (University Arizona Press), 767

Ormel, C. W., Spaans, M., \& Tielens, A. G. G. M. 2007, A\&A, 461, 215

Prokopjeva, M. S., \& Il'in, V. B. 2007, Astron. Lett., 33, 699

Rouleau, F., \& Martin, P. G. 1991, ApJ, 377, 526

Sargent, B., Forrest, W. J., D’Alessio, P., et al. 2006, ApJ, 645, 395

Schegerer, A., Wolf, S., Voshchinnikov, N. V., Przygodda, F., \& Kessler-Silacci, J. E. 2006, A\&A, 456, 535

Shen, Y., Draine, B. T., \& Johnson, E. T. 2008, ApJ, in press [arXiv: 0801.1996]

Sicilia-Aguilar, A., Hartman, L. W., Watson, D., et al. 2007, ApJ, 659, 1637

van Boekel, R., Min, M., Waters, L. B. F. M., et al. 2005, A\&A, 437, 189

Voshchinnikov, N. V., \& Mathis, J. S. 1999, ApJ, 526, 257

Voshchinnikov, N. V., Il'in, V. B., \& Henning, Th. 2005, A\&A, 429, 371

Voshchinnikov, N. V., Il'in, V. B., Henning, Th., \& Dubkova, D. N. 2006, A\&A, 445,167

Watson, D. M., Leisenring, J. M., Furlan, E., et al. 2007, ApJS, in press [arXiv: 0704.1518] 
N. V. Voshchinnikov and T. Henning: Is the silicate emission feature only influenced by grain size?, Online Material p 1

Table 1. Grain porosity and fractional amount of carbon in grains as derived from fitting the $10 \mu \mathrm{m}$ silicate emission feature.

\begin{tabular}{|c|c|c|c|c|c|c|c|}
\hline Star & Type $^{1}$ & $\mathcal{P}$ & $V_{\mathrm{Bel} 1} / V_{\text {solid }}$ & $M_{\mathrm{Be} 1} / M_{\mathrm{MgFeSiO}_{4}}$ & Age, Myr & Ref. $^{2}$ & Comment \\
\hline Tr 37 73-758 & $\mathrm{t}$ & 0.784 & 0.259 & 0.196 & 1.8 & 1 & \\
\hline Tr $3711-2146$ & $\mathrm{t}$ & 0.705 & 0.217 & 0.156 & 0.9 & 1 & \\
\hline Tr $3711-2037$ & $\mathrm{t}$ & 0.615 & 0.232 & 0.170 & 2.5 & 1 & \\
\hline $\operatorname{Tr} 3711-2031$ & $\mathrm{t}$ & 0.542 & 0.167 & 0.113 & 2.5 & 1 & \\
\hline Tr 37 14-183 & $\mathrm{t}$ & 0.704 & 0.281 & 0.219 & 0.9 & 1 & \\
\hline $\operatorname{Tr} 3782-272$ & $\mathrm{t}$ & 0.726 & 0.188 & 0.130 & 10.5 & 1 & \\
\hline $\operatorname{Tr} 37$ 12-2113 & $\mathrm{t}$ & 0.650 & 0.186 & 0.128 & 1.1 & 1 & \\
\hline Tr 37 13-157 & $\mathrm{t}$ & 0.804 & 0.250 & 0.187 & 2.4 & 1 & \\
\hline $\operatorname{Tr} 37$ 91-155 & $\mathrm{t}$ & 0.976 & 0.320 & 0.264 & 1.7 & 1 & \\
\hline Tr 37 54-1547 & $\mathrm{t}$ & 0.556 & 0.132 & 0.085 & 5.7 & 1 & \\
\hline Tr 37 13-1250 & $\mathrm{t}$ & 0.649 & 0.124 & 0.079 & 3.3 & 1 & \\
\hline Tr 37 23-162 & $\mathrm{t}$ & 0.722 & 0.247 & 0.184 & 6.6 & 1 & \\
\hline $\operatorname{Tr} 3701-580$ & $\mathrm{t}$ & 0.604 & 0.167 & 0.112 & 8.7 & 1 & \\
\hline NGC 7160 DG-481 & $\mathrm{h}$ & 0.852 & 0.333 & 0.279 & 12.0 & 1 & \\
\hline SU Aur & $\mathrm{t}$ & 0.731 & 0.131 & 0.085 & 3.9 & 2 & age from $(5)$ \\
\hline GW Ori & $\mathrm{t}$ & 0.562 & 0.102 & 0.064 & 1.0 & 2 & \\
\hline CR Cha & $\mathrm{t}$ & 0.471 & 0.107 & 0.067 & 1.0 & 2 & \\
\hline Glass I & $\mathrm{t}$ & 0.671 & 0.208 & 0.147 & 1.0 & 2 & \\
\hline WW Cha & $\mathrm{t}$ & 0.539 & 0.194 & 0.135 & 0.3 & 2 & \\
\hline SZ 82 & $\mathrm{t}$ & 0.427 & 0.376 & 0.338 & 1.1 & 2 & \\
\hline AS 205S & $\mathrm{t}$ & 0.556 & 0.251 & 0.188 & 0.1 & 2 & \\
\hline Haro 1-16 & $\mathrm{t}$ & 0.425 & 0.141 & 0.092 & 0.5 & 2 & \\
\hline AK Sco & $\mathrm{t}$ & 0.519 & 0.116 & 0.073 & 7.6 & 2 & age from (5) \\
\hline FM Tau & $\mathrm{t}$ & 0.598 & 0.256 & 0.193 & 2.8 & 3 & age from (6) \\
\hline GG Tau A & $\mathrm{t}$ & 0.401 & 0.356 & 0.310 & 3.3 & 3 & age from (7) \\
\hline GG Tau B & $\mathrm{t}$ & 0.841 & 0.342 & 0.292 & 1.6 & 3 & age from (7) \\
\hline GM Aur & $\mathrm{t}$ & 0.700 & 0.162 & 0.109 & 7.4 & 3 & age from (7) \\
\hline IP Tau & $\mathrm{t}$ & 0.493 & 0.213 & 0.152 & 4.3 & 3 & age from (7) \\
\hline TW Hya & $\mathrm{t}$ & 0.756 & 0.158 & 0.105 & 10.0 & 3 & age from (2) \\
\hline Hen 3-600 A & $\mathrm{t}$ & 0.860 & 0.239 & 0.176 & & 3 & \\
\hline V410 Anon 13 & $\mathrm{t}$ & 0.651 & 0.398 & 0.370 & & 3 & \\
\hline HD 104237 & $\mathrm{~h}$ & 0.612 & 0.307 & 0.249 & 4.8 & 4 & age from (5) \\
\hline HD 142527 & $\mathrm{~h}$ & 0.673 & 0.301 & 0.242 & 1.0 & 4 & \\
\hline HD 142666 & $\mathrm{~h}$ & 0.680 & 0.268 & 0.205 & 4.4 & 4 & age from (5) \\
\hline HD 144432 & $\mathrm{~h}$ & 0.440 & 0.123 & 0.078 & 6.65 & 4 & age from (5) \\
\hline HD 144668 & $\mathrm{~h}$ & 0.832 & 0.504 & 0.569 & 0.5 & 4 & \\
\hline HD 150193 & h & 0.398 & 0.180 & 0.124 & 2.6 & 4 & age from (5) \\
\hline HD 163296 & $\mathrm{~h}$ & 0.537 & 0.209 & 0.148 & 6.0 & 4 & age from (5) \\
\hline HD 179218 & $\mathrm{~h}$ & 0.948 & 0.292 & 0.231 & 1.3 & 4 & \\
\hline HD 245185 & $\mathrm{~h}$ & 0.704 & 0.149 & 0.098 & 3.3 & 4 & age from (5) \\
\hline HD 36112 & $\mathrm{~h}$ & 0.431 & 0.122 & 0.078 & 4.4 & 4 & age from (5) \\
\hline HD 37357 & $\mathrm{~h}$ & 0.480 & 0.206 & 0.146 & 10.0 & 4 & \\
\hline HD 37806 & $\mathrm{~h}$ & 0.732 & 0.405 & 0.382 & 0.8 & 4 & \\
\hline AB Aur & $\mathrm{h}$ & 0.714 & 0.187 & 0.129 & 4.8 & 4 & age from (7) \\
\hline HK Ori & $\mathrm{h}$ & 0.551 & 0.400 & 0.374 & 5.8 & 4 & age from (5) \\
\hline UX Ori & $\mathrm{h}$ & 0.392 & 0.160 & 0.107 & 2.8 & 4 & age from (5) \\
\hline V380 Ori & $\mathrm{h}$ & 0.694 & 0.493 & 0.545 & 7.4 & 4 & age from (5) \\
\hline
\end{tabular}

${ }^{1}$ Meaning of symbols: $\mathrm{t}=\mathrm{T}$ Tau star, $\mathrm{h}=$ Herbig Ae/Be star.

2 References for observational data: (1) Sicilia-Aguilar et al. (2007); (2) Schegerer et al. (2006); (3) Sargent et al. (2006);

(4) van Boekel et al. (2005); (5) Blondel \& Tjin A Djie (2006); (6) Güdel et al. (2007); (7) Bertout et al. (2007). 